\title{
Recent advances in polyubiquitin chain recognition
}

\section{Hao Wu*, Yu-Chih Lo and Su-Chang Lin}

\author{
Address: Department of Biochemistry, Weill Cornell Medical College, 1300 York Avenue, New York, NY 10021, USA \\ *Corresponding author: Hao Wu (haowu@med.cornell.edu) \\ FI000 Biology Reports 2010, 2:20 (doi:10.34I0/B2-20) \\ The electronic version of this article is the complete one and can be found at: http://fl000.com/reports/biology/content/2/20
}

\begin{abstract}
Polyubiquitin chains are regulatory signals for a wide array of biological processes. Recent structural studies reveal novel modes of polyubiquitin chain recognition and implicate the diverse repertoire of interactions in providing the specificity of polyubiquitin recognition.
\end{abstract}

\section{Introduction and context}

As the name implies, ubiquitin (residues 1-76) is a ubiquitous signaling molecule for a wide array of biological processes, including protein degradation, assembly of signaling complexes, and DNA damage repair. When attached to proteins, ubiquitin or polyubiquitin chains act as 'codes' to direct suitable proteinprotein interactions to effect or regulate specific cellular functions. Ubiquitin is conjugated to target proteins or itself through an isopeptide linkage between the side chain amino group of lysine (Lys) residues and the C-terminal carboxyl group of ubiquitin. This process is achieved by the sequential action of ubiquitin-activating enzymes (E1), ubiquitin-conjugating enzymes (E2), and ubiquitin ligases (E3). The ubiquitin 'codes' on cellular proteins can be monoubiquitin, multiple monoubiquitins, or polyubiquitin chains of diverse length and linkage. There are seven Lys residues on ubiquitins (Lys6, Lys11, Lys27, Lys29, Lys33, Lys48, and Lys63), allowing for seven possible homotypic linkages and multiple possible mixed chains. The best characterized polyubiquitin linkage types are those mediated by Lys 48 and Lys63, with the former known in targeting proteins for proteasomal degradation and the latter involved in multiple signaling processes, in particular nuclear factorkappa-B (NF- $\kappa$ B) activation. Recent studies identified a new linkage type of polyubiquitin chains: the linear linkage formed between the N-terminal amino group and the C-terminal carboxyl group (see 'Interactions of NEMO with diubiquitins: both revelations and confusions' section below). Like phosphorylation, ubiquitination is highly reversible through the action of deubiquitinating enzymes (DUBs) and therefore acts as a transient and versatile signaling element.

Ubiquitin recognition by the collective entity of ubiquitin-binding domains (UBDs) is at the center of ubiquitin-mediated signaling. So far, investigators have discovered over 20 types of UBDs, such as ubiquitinassociated domains (UBAs), ubiquitin-interacting motifs (UIMs), zinc fingers (ZFs), and Jab1/MPN domains for the metalloprotease class of DUBs. Monoubiquitin recognition has been extensively studied and reviewed elsewhere [1,2], however, only limited examples of polyubiquitin recognition were known until very recently. Here, we will summarize recent advances in understanding the molecular basis of diubiquitin recognition and, in some cases, the extrapolation to longer polyubiquitin chains.

\section{Major recent advances}

A very first example of diubiquitin recognition is provided by the nuclear magnetic resonance (NMR)derived structure of Lys48-linked diubiquitin in complex with a UBA domain of hHR23A, a protein implicated in the modulation of polyubiquitin interaction with the proteasome [3]. In this structure, the distal and the proximal ubiquitin molecules use the conserved hydrophobic patch centered on residue Ile44 to grip onto either side of the UBA domain, which consists of a compact triple-helical bundle (Figure 1A). In a diubiquitin, the distal ubiquitin provides the $\mathrm{C}$-terminal tail 
Figure I. Structures of ubiquitin-binding domain (UBD)-diubiquitin complexes

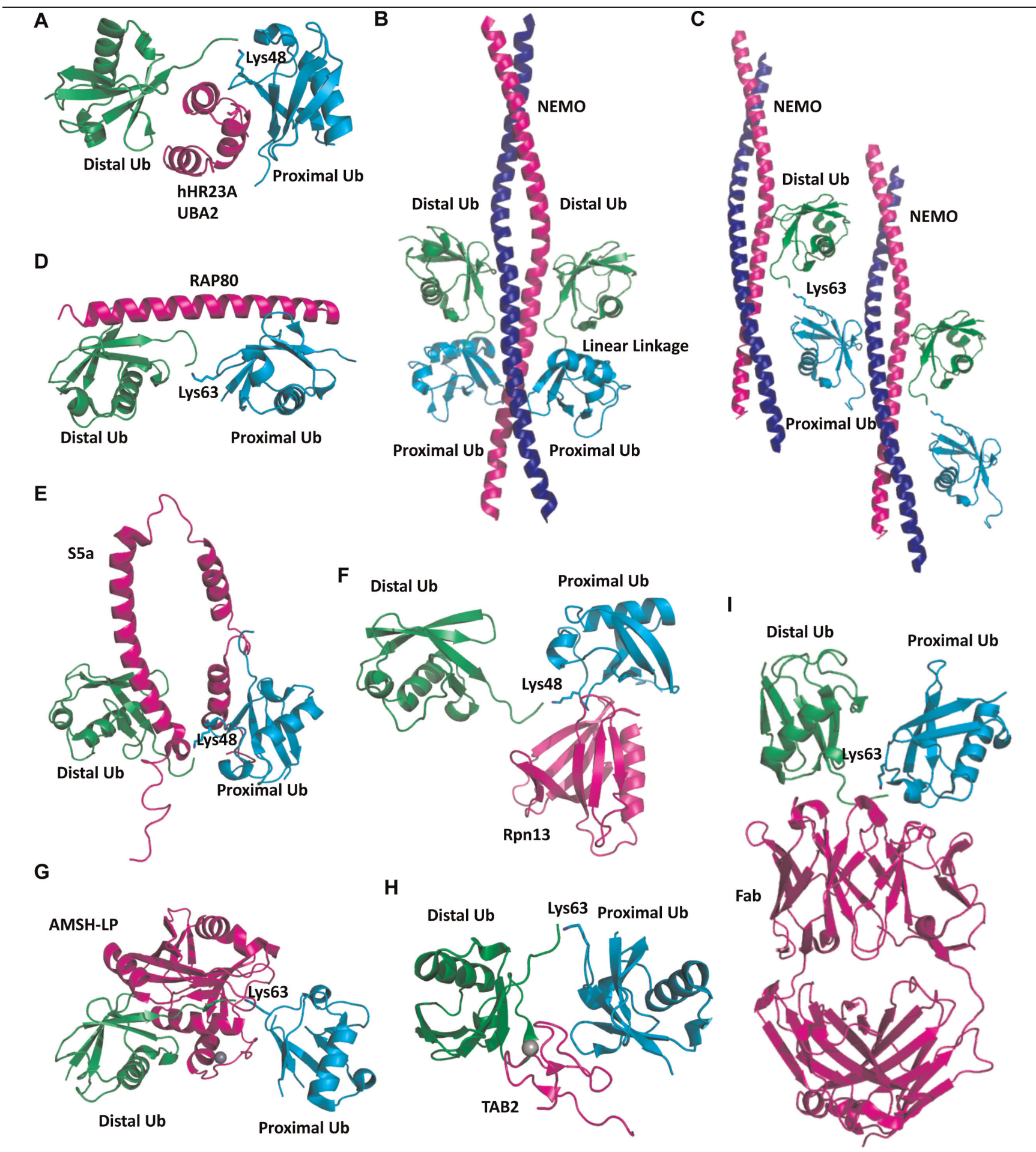

All distal and proximal ubiquitins are shown in green and cyan, respectively. The UBDs are shown in magenta. The Lys 48 and Lys 63 side chains at the linkages are shown as stick models and are labeled. Linear linkage is shown as a continuous polypeptide and is labeled. (A) The hHR23A-Lys48 diubiquitin complex. (B) The NEMO-linear diubiquitin complex. (C) The NEMO-Lys63 diubiquitin complex. Two-unit cell contents are shown to illustrate the interaction at the distal ubiquitin with one NEMO molecule and the interaction at the proximal ubiquitin with another NEMO molecule. (D) The RAP80-Lys63 diubiquitin complex. (E) The S5a-Lys48 diubiquitin complex. (F) The Rpn I3-Lys48 diubiquitin complex. (G) The AMSH-LP-Lys63 diubiquitin complex. (H) The TAB2Lys63 diubiquitin complex. (I) An antibody Fab fragment-Lys63 diubiquitin complex. Lys, lysine; Ub, ubiquitin. 
while the proximal ubiquitin provides the amino group for the isopeptide bond. The past year or so has seen an explosion of structural information on the recognition of Lys63-linked or Lys48-linked or linear diubiquitins. The new structures of UBD-diubiquitin complexes from different cellular processes provide an expanded view of the diversity of such interactions.

\section{Interactions of NEMO with diubiquitins: both revelations and confusions}

NEMO is the regulatory subunit of the IKK (I $\kappa$ B kinase) complex for NF-KB activation. Recent studies have shown that the UBAN (ubiquitin binding in ABIN [A20-binding inhibitor of NF- $\kappa \mathrm{B}$ activation] and NEMO [NF- $\kappa \mathrm{B}$ essential modulator]) domain directly interacts with linear polyubiquitin chains [4-7]. The UBAN motif of NEMO forms a dimeric coiled coil and uses both chains for linear diubiquitin interactions. Structure-based mutagenesis and NMR studies revealed that the Ile44 patch and the C-terminal tail of the distal ubiquitin in linear diubiquitin are most critical for UBAN interaction [6]. The crystal structure of the UBAN-linear diubiquitin complex revealed interfaces that are similar to the NMRbased studies [7]. However, despite the measured stoichiometry in solution of one UBAN dimer per linear diubiquitin $[6,8]$, the crystal structure showed that two dibubiquitin molecules interact symmetrically on each side of the UBAN dimer [7] (Figure 1B). It is possible that only one side of the dimeric UBAN is bound with diubiquitin in solution and that the other side was forced to interact with another diubiquitin molecule under the crystallization condition. Consistent with this assessment, it appears that at high diubiquitin concentrations, there is a second, weaker binding site [8].

The UBAN motif of NEMO also directly interacts with Lys63-linked polyubiquitin [4-6,9], albeit at a much lower affinity than with linear diubiquitin [6]. Similar mutagenesis and NMR-based methods revealed that the Ile44 patch of the proximal ubiquitin in Lys63-linked diubiquitin and the $\mathrm{C}$-terminal tail of the distal ubiquitin provide the critical binding energy for the UBAN interaction [6]. In addition, the stoichiometry of the interaction was shown to be one UBAN dimer per Lys63-linked diubiquitin [6]. In contrast to the crystal structure of the UBAN-linear diubiquitin complex [7], the crystal structure of the UBAN-Lys63-linked diubiquitin complex showed that the diubiquitin interacts with only one side of the UBAN dimer [9] (Figure 1C). In fact, only one ubiquitin molecule in the diubiquitin, either the proximal or the distal ubiquitin, interacts with UBAN in the crystal lattice, and this is different from the higher energetic role of the proximal ubiquitin shown by mutagenesis experiments in solution [6].
Recent NMR and cellular studies showed that the C-terminal ZF of NEMO is also a functional UBD and plays critical roles in NF- $\kappa B$ activation [10]. Remarkably, while neither the UBAN nor the ZF domains shows any preference for K63-linked polyubiquitin chains, they together form a bipartite high-affinity K63-specific UBD [11]. Further structural studies are required to reveal the molecular basis for this specificity.

Recognition of Lys63-linked diubiquitin by UIMs of RAP80 Receptor-associated protein 80 (RAP80) plays a key role in recruiting the Abraxas-BRCC36-BRCA1-BARD1 complex to DNA damage foci for DNA repair by possessing an Abraxas-interacting region. It also contains tandem UIMs, which are single-helical motifs, for interaction with Lys63-linked polyubiquitin. The crystal structure of the tandem UIMs of RAP80 in complex Lys63-linked diubiquitin revealed that the two UIMs of RAP80 and the inter-UIM region form a continuous helix $[12,13]$. The proximal and the distal ubiquitins of the Lys63-linked diubiquitin interact with UIM1 and UIM2, respectively, in a manner that involves the Ile44 patches (Figure 1D). The specific spacing between the UIMs appears to be critical in the specificity of the interaction for Lys63 linkage, although no direct interaction is present between RAP80 and the Lys63-isopeptide bond $[12,13]$.

\section{Concerted polyubiquitin interactions by ubiquitin receptors in the proteasome}

Lys48-linked polyubiquitin recognitions by the two ubiquitin receptors in the proteasome, S5a and Rpn13, have been revealed by elegant crystallographic and NMR studies $[14,15]$. S5a contains two independent UIMs and its UIM2 preferentially interacts with the proximal ubiquitin in a Lys48-linked diubiquitin (Figure 1E). Rpn13 contains a pleckstrin-like receptor for ubiquitin (Pru) domain and uses loops rather than secondary structural elements for ubiquitin interaction [14]. Dibuqiutin recognition by Rpn13 is mediated mostly by binding to the proximal ubiquitin at the Ile 44 patch and to the Lys48 linkage between the proximal and the distal ubiquitin (Figure 1F). It is proposed that in the intact proteasome, Rpn13 and S5a interact simultaneously with tetraubiquitin or a longer polyubiquitin to accomplish polyubiquitin targeting to the proteasome [15].

\section{Specificity for different linkages in DUBs}

AMSH-like protease (AMSH-LP) is a Jab1/MPN domaincontaining DUB with a Zn-coordinating catalytic core. The crystal structure of Lys63-linked diubiquitin in complex with AMSH-LP revealed that the distal ubiquitin uses the Ile44 hydrophobic patch for interaction with AMSH-LP while the proximal ubiquitin uses an alternative surface [16]. Most strikingly, the surface of AMSH-LP forms a 
catalytic groove that accommodates the Lys63 side chain of the proximal ubiquitin and the isopeptide-linked C-terminal tail of the distal ubiquitin (Figure 1G). Only the Lys63-linked proximal ubiquitin can bind in the proper orientation for catalysis. Sequence similarity also suggests that the specificity of AMSH for Lys63-linked chains is derived primarily from the interactions with the proximal ubiquitin. Bidentate recognition of both proximal and distal ubiquitins has also been shown as the mechanism of specificity for K48 linkage in the ovarian tumor domain-containing DUB, Otubain 1 [17].

\section{Specific recognition of Lys63-linked diubiquitin conformation by TAB2 and TAB3}

Activation of the kinase TAK1 in JNK and NF- $\mathrm{BB}$ signaling pathways requires interaction with Lys63linked polyubiquitin chains by the TAK1-binding proteins, TAB2 and TAB3. TAB2 and TAB3 recognize Lys63-linked chains, but not linear chains, using their C-terminal Np14 zinc finger (NZF) domains. Crystal structures of the TAB2 NZF domain in complex with Lys63-linked diubiquitin or triubiquitin and the TAB3 NZF domain in complex with Lys63-linked diubiquitin reveal that each NZF domain interacts with a diubiquitin using two distinct binding sites and that multiple NZF domains could recognize longer polyubiquitin chains successively [18,19] (Figure 1H). The NZF domains recognize the Ile44 hydrophobic patches of both the proximal and the distal ubiquitins in a diubiquitin. Interestingly, the Lys63-linked isopeptide bond is not involved in the interaction. In contrast, the specificity of the interaction for Lys63-linked chains versus linear chains is conformational as the Met1 residue of the proximal ubiquitin is too far away from the C-terminus of the distal ubiquitin in the diubiquitin complexes with the NZF of TAB2 and TAB3.

\section{Recognition of Lys63-linked diubiquitin by a specific antibody}

Interestingly, in an effort to reveal the role of polyubiquitin chains of specific linkages in diverse biological processes, antibodies were developed to recognize Lys63 versus Lys48 linkages [20]. The crystal structure of a Fab fragment of a specific antibody in complex with Lys63linked diubiquitin revealed that the Fab interacts with the diubiquitin at either side of the isopeptide linkage with limited involvement of the Ile44 patch of either ubiquitin (Figure 1I). It appears that the Fab specifically recognizes a conformation of the diubiquitin that is compatible with the Lys63, but not the Lys48, linkage.

\section{Future directions}

The recent structural studies may have pointed out a number of rules or the lack of them in diubiquitin recognition. First of all, diverse classes of UBDs are capable of diubiquitin recognition and achieve the specificity in their own manners. Second, the Ile44 hydrophobic patch is often crucial for diubiquitin recognition, as is the case for monoubiquitin interaction, and the spatial separation of UBDs in a protein may facilitate the simultaneous interaction with the Ile 44 patches in a diubiquitin. Third, the linkage residues themselves may or may not be directly involved in UBD interaction. Fourth, and importantly, intrinsic conformational preferences in solution of polyuibiquitin chains of different linkages are important determinants in the specificity of polyubiquitin chain recognition. Structural studies of diubiquitins and tetraubiquitins have revealed that Lys48-linked chains in general prefer a more compact structure than either Lys63-linked chains or linear chains [21-27]. It should be cautioned, however, that crystal packing exerts significant influences in polyubiquitin chain conformation $[25,27]$. Overall, it appears that nature has developed a diverse repertoire of interactions to provide specificity of polyubiquitin recognition in biological processes.

Future structural studies on polyubiquitin chain interaction may reveal recognition of UBDs with longer-chain polyubiquitins. It is known that diubiquitin is often not sufficient for eliciting the intended biological responses such as in proteasomal targeting and NF-KB activation. One reason for this requirement has been demonstrated for simultaneous polyubiquitin chain recognition by two UBDs, which sets the chain length threshold [28]. Similarly, long polyubiquitin chains may serve as extended scaffolds to mediate recruitment of multiple copies of the signaling proteins to induce oligomerization. Another reason for the requirement of longer chains may be avidity, which increases the strength of the interactions. Studies on long polyubiquitin chains may be challenging but will certainly transform our current thinking in polyubiquitin signaling.

\section{Abbreviations}

AMSH-LP, AMSH-like protease; DUB, deubiquitinating enzyme; Lys, lysine; NEMO, NF- $\kappa \mathrm{B}$ essential modulator; NF- $\kappa B$, nuclear factor-kappa-B; NMR, nuclear magnetic resonance; NZF, Np14 zinc finger; RAP80, receptorassociated protein 80; UBA, ubiquitin-associated domain; UBAN, ubiquitin binding in ABIN (A20-binding inhibitor of NF- $\kappa B$ activation) and NEMO (NF- $\kappa B$ essential modulator); UBD, ubiquitin-binding domain; UIM, ubiquitin-interacting motif; ZF, zinc finger.

\section{Competing interests}

The authors declare that they have no competing interests. 


\section{Acknowledgments}

The work was supported by a National Institutes of Health grant (R01AI079260) to HW.

\section{References}

I. Hurley JH, Lee S, Prag G: Ubiquitin-binding domains. Biochem J 2006, 399:36I-72.

2. Dikic I, Wakatsuki S, Walters KJ: Ubiquitin-binding domains from structures to functions. Nat Rev Mol Cell Biol 2009, |0:659-7|.

3. Varadan R, Assfalg M, Raasi S, Pickart C, Fushman D: Structural determinants for selective recognition of a Lys48-linked polyubiquitin chain by a UBA domain. Mol Cell 2005, I 8:687-98.

FI000 Factor 6.0 Must Read

Evaluated by Jane Endicott 27 Jun 2005

4. Ea CK, Deng L, Xia ZP, Pineda G, Chen ZJ: Activation of IKK by TNFalpha requires site-specific ubiquitination of RIPI and polyubiquitin binding by NEMO. Mol Cell 2006, 22:245-57.

FI000 Factor 3.0 Recommended Evaluated by Neal Silverman I5 May 2006

5. Wu CJ, Conze DB, Li T, Srinivasula SM, Ashwell JD: Sensing of Lys 63-linked polyubiquitination by NEMO is a key event in NF-kappaB activation [corrected]. Nat Cell Biol 2006, 8:398-406.

FI000 Factor 4.8 Must Read

Evaluated by Neal Silverman 15 May 2006, Jurg Tschopp 22 Mar 2006

6. Lo YC, Lin SC, Rospigliosi CC, Conze DB, Wu Cl, Ashwell JD, Eliezer D, Wu H: Structural basis for recognition of diubiquitins by NEMO. Mol Cell 2009, 33:602-I5.

7. Rahighi S, Ikeda F, Kawasaki M, Akutsu M, Suzuki N, Kato R, Kensche T, Uejima T, Bloor S, Komander D, Randow F, Wakatsuki S, Dikic I: Specific recognition of linear ubiquitin chains by NEMO is important for NF-kappaB activation. Cell 2009, 136: 1098-109.

8. Ivins FJ, Montgomery MG, Smith SJ, Morris-Davies AC, Taylor IA, Rittinger K: NEMO oligomerisation and its ubiquitin-binding properties. Biochem J 2009, 42 I:243-5I.

FI000 Factor 3.0 Recommended

Evaluated by Ivan Dikic 2I May 2009

9. Yoshikawa A, Sato Y, Yamashita M, Mimura H, Yamagata A, Fukai S: Crystal structure of the NEMO ubiquitin-binding domain in complex with Lys 63-linked di-ubiquitin. FEBS Lett 2009, 583:3317-22.

10. Cordier F, Grubisha O, Traincard F, Véron M, Delepierre M, Agou F: The zinc finger of NEMO is a functional ubiquitin-binding domain. J Biol Chem 2009, 284:2902-7.

II. Laplantine E, Fontan E, Chiaravalli J, Lopez T, Lakisic G, Véron M, Agou F, Israël A: NEMO specifically recognizes K63-linked poly-ubiquitin chains through a new bipartite ubiquitinbinding domain. EMBO J 2009, 28:2885-95.

12. Sims JJ, Cohen RE: Linkage-specific avidity defines the lysine 63-linked polyubiquitin-binding preference of rap80. Mol Cell 2009, 33:775-83.

FI000 Factor 3.0 Recommended

Evaluated by Zhen-Qiang Pan 23 Apr 2009

13. Sato Y, Yoshikawa A, Mimura H, Yamashita M, Yamagata A, Fukai S: Structural basis for specific recognition of Lys 63-linked polyubiquitin chains by tandem UIMs of RAP80. EMBO J 2009, 28:246I-8.
14. Schreiner P, Chen X, Husnjak K, Randles L, Zhang N, Elsasser S, Finley D, Dikic I, Walters KJ, Groll M: Ubiquitin docking at the proteasome through a novel pleckstrin-homology domain interaction. Nature 2008, 453:548-52.

15. Zhang N, Wang Q, Ehlinger A, Randles L, Lary JW, Kang Y, Haririnia A, Storaska AJ, Cole JL, Fushman D, Walters KJ: Structure of the s5a:k48-linked diubiquitin complex and its interactions with rpn I3. Mol Cell 2009, 35:280-90.

16. Sato Y, Yoshikawa A, Yamagata A, Mimura H, Yamashita M, Ookata K, Nureki O, Iwai K, Komada M, Fukai S: Structural basis for specific cleavage of Lys 63-linked polyubiquitin chains. Nature 2008 , 455:358-62.

17. Wang T, Yin L, Cooper EM, Lai MY, Dickey S, Pickart CM, Fushman D, Wilkinson KD, Cohen RE, Wolberger C: Evidence for bidentate substrate binding as the basis for the K48 linkage specificity of otubain I. J Mol Biol 2009, 386:10 II-23.

18. Kulathu Y, Akutsu M, Bremm A, Hofmann K, Komander D: Two-sided ubiquitin binding explains specificity of the TAB2 NZF domain. Nat Struct Mol Biol 2009, 16:1328-30.

19. Sato Y, Yoshikawa A, Yamashita M, Yamagata A, Fukai S: Structural basis for specific recognition of Lys 63 -linked polyubiquitin chains by NZF domains of TAB2 and TAB3. EMBO J 2009, 28:3903-9.

FI000 Factor 3.0 Recommended

Evaluated by Jane Endicott 12 Aug 2009

20. Newton K, Matsumoto ML, Wertz IE, Kirkpatrick DS, Lill JR, Tan J, Dugger D, Gordon N, Sidhu SS, Fellouse FA, Komuves L, French DM, Ferrando RE, Lam C, Compaan D, Yu C, Bosanac I, Hymowitz SG, Kelley RF, Dixit VM: Ubiquitin chain editing revealed by polyubiquitin linkage-specific antibodies. Cell 2008, I34:668-78.

FI000 Factor 4.8 Must Read

Evaluated by Ze'ev Ronai 08 Sep 2008, Paolo Rossi 22 Sep 2008

21. Varadan R, Walker O, Pickart C, Fushman D: Structural properties of polyubiquitin chains in solution. J Mol Biol 2002, 324:637-47.

FI000 Factor 3.0 Recommended

Evaluated by Jane Endicott 19 Mar 2004

22. Komander D, Reyes-Turcu F, Licchesi JD, Odenwaelder P, Wilkinson KD, Barford D: Molecular discrimination of structurally equivalent Lys 63 -linked and linear polyubiquitin chains. EMBO Rep 2009, 10:466-73.

23. Varadan R, Assfalg M, Haririnia A, Raasi S, Pickart C, Fushman D: Solution conformation of Lys63-linked di-ubiquitin chain provides clues to functional diversity of polyubiquitin signaling. J Biol Chem 2004, 279:7055-63.

FI000 Factor 3.0 Recommended

Evaluated by Jane Endicott 19 Mar 2004

24. Cook WJ, Jeffrey LC, Kasperek E, Pickart CM: Structure of tetraubiquitin shows how multiubiquitin chains can be formed. J Mol Biol 1994, 236:601-9.

25. Datta $A B$, Hura GL, Wolberger $C$ : The structure and conformation of Lys63-linked tetraubiquitin. J Mol Biol 2009, 392: I I I 7-24.

26. Phillips CL, Thrower J, Pickart CM, Hill CP: Structure of a new crystal form of tetraubiquitin. Acta Crystallogr D Biol Crystallogr 200I, 57(Pt 2):34I-4.

27. Eddins MJ, Varadan R, Fushman D, Pickart CM, Wolberger C: Crystal structure and solution NMR studies of Lys48-linked tetraubiquitin at neutral pH. J Mol Biol 2007, 367:204-II.

28. Zhang D, Chen T, Ziv I, Rosenzweig R, Matiuhin Y, Bronner V, Glickman MH, Fushman D: Together, Rpn 10 and Dsk2 can serve as a polyubiquitin chain-length sensor. Mol Cell 2009, 36: $1018-33$. 